\title{
TFCrowd: A Blockchain-based Crowdsourcing Framework with Enhanced Trustworthiness and Fairness
}

\section{Chunxiao Li}

Beijing Normal University

\section{Xidi Qu}

Beijing Normal University

Yu Guo ( $\nabla$ yuguo@bnu.edu.cn )

Beijing Normal University https://orcid.org/0000-0002-9078-7462

\section{Research}

Keywords: Crowdsourcing, Repuatation, Shapley value, Smart contract, Blockchain

Posted Date: February 1st, 2021

DOl: https://doi.org/10.21203/rs.3.rs-154519/v1

License: (c) (i) This work is licensed under a Creative Commons Attribution 4.0 International License. Read Full License

Version of Record: A version of this preprint was published at EURASIP Journal on Wireless Communications and Networking on August 19th, 2021. See the published version at https://doi.org/10.1186/s13638-021-02040-z. 


\section{Abstract}

Blockchain technology has attracted considerable attention due to the boom of cryptocurrencies and decentralized applications. Among them, the emerging blockchain-based crowdsourcing is a typical paradigm, which gets rid of centralized cloud-servers and leverages smart contracts to realize task recommendation and reward distribution. However, there are still two critical issues yet to be solved urgently. First, malicious evaluation from crowdsourcing requesters will result in honest workers not getting the rewards they deserve even providing valuable solutions. Second, unfair evaluation and reward distribution can lead to workers' reluctance to work actively. Therefore, the above problems will seriously hinder the development of blockchain-based crowdsourcing platforms.

In this paper, we propose a new blockchain-based crowdsourcing framework with enhanced trustworthiness and fairness, named TFCrowd. The core idea of TFCrowd is utilizing a smart contract of blockchain as a trusted authority to fairly evaluate contributions and allocate rewards. To this end, we devise a reputation-based evaluation mechanism to punish the requester who behaves as "falsereporting" and a Shapley value-based method to distribute rewards fairly. By using our proposed schemes, TFCrowd can prevent malicious requesters from making unfair comments and reward honest workers according to their contributions. Extensive simulations and the experiment results demonstrate that TFCrowd can protect the interests of workers and distribute rewards fairly.

\section{Full Text}

Due to technical limitations, full-text HTML conversion of this manuscript could not be completed. However, the manuscript can be downloaded and accessed as a PDF.

\section{Figures}




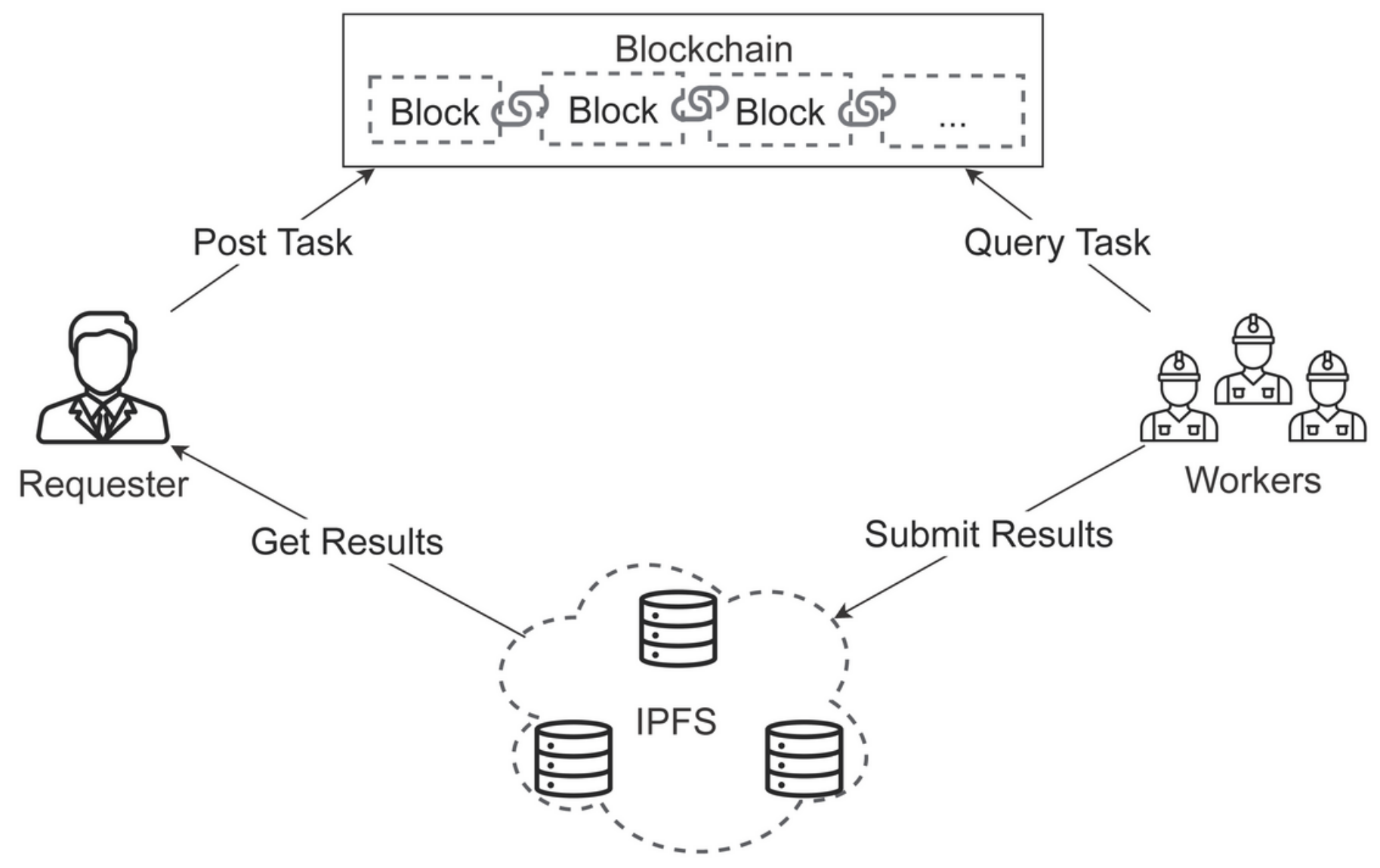

Figure 1

Overview of TFCrowd architecture. 


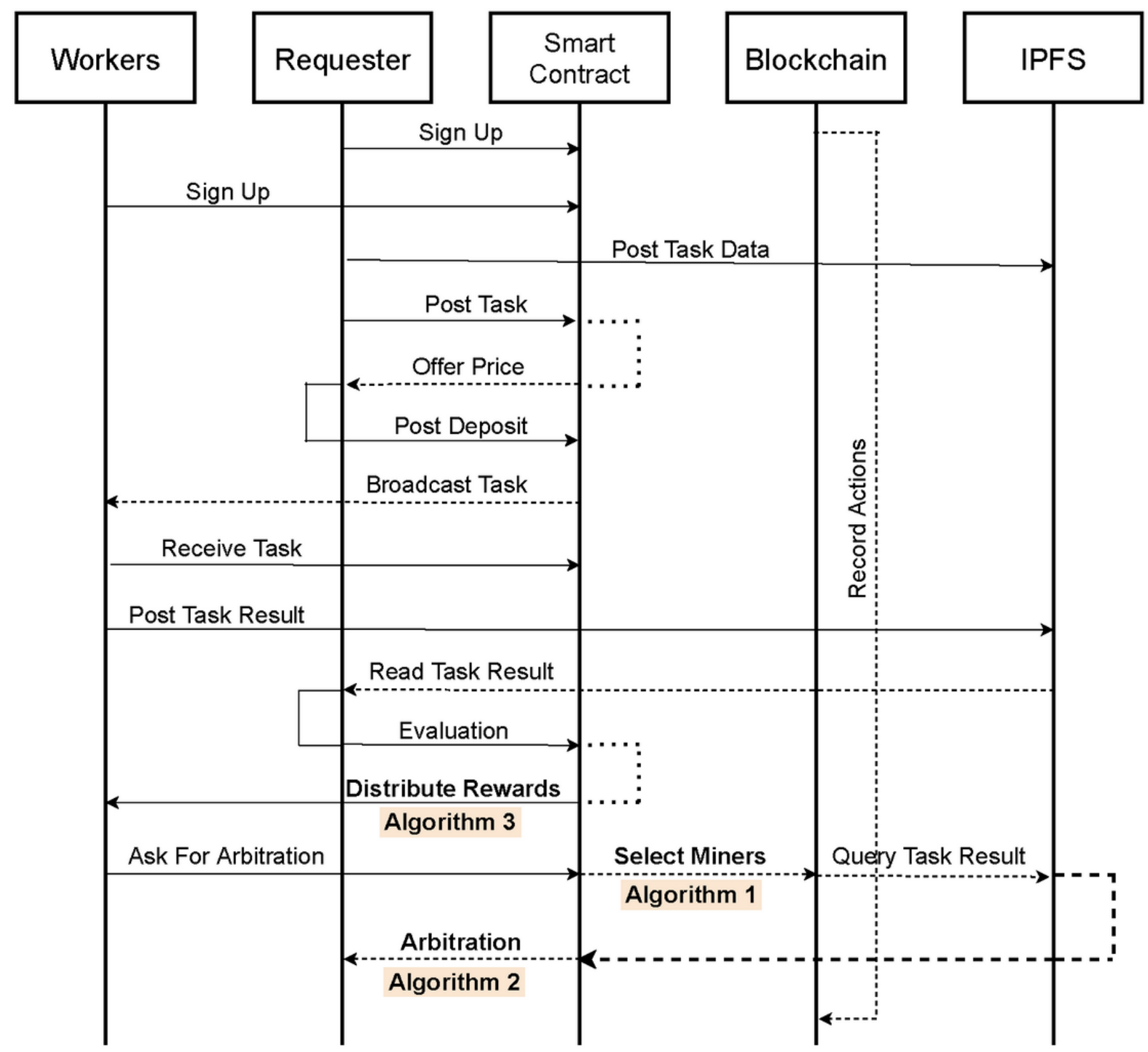

Figure 2

Workflow of TFCrowd. 
Register

Application
Task

Application
Pay

Application
Arbitration

Application

\section{Application Layer}

\begin{tabular}{c|c|c|c|}
\hline User & Task & Reputation & Arbitration \\
Management & Management & Mangement & Mangement \\
Smart Contract & Smart Contract & Smart Contract & Smart Contract \\
\hline
\end{tabular}

Program Environment

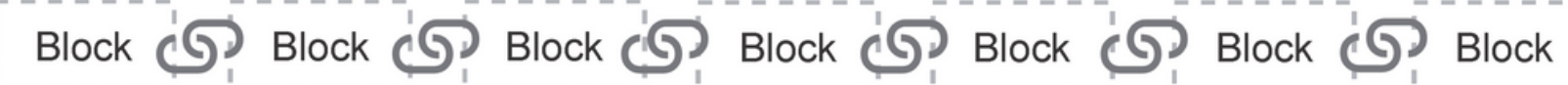
Blocḱnain Layer

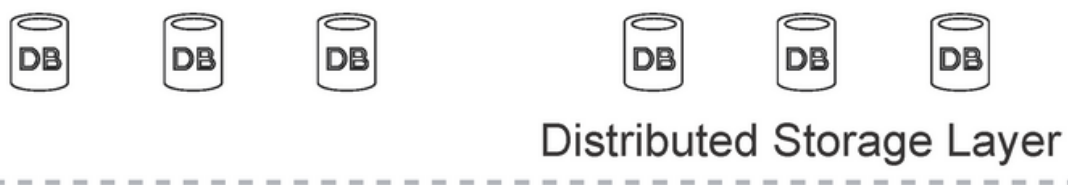

Figure 3

Logical structure of TFCrowd. 


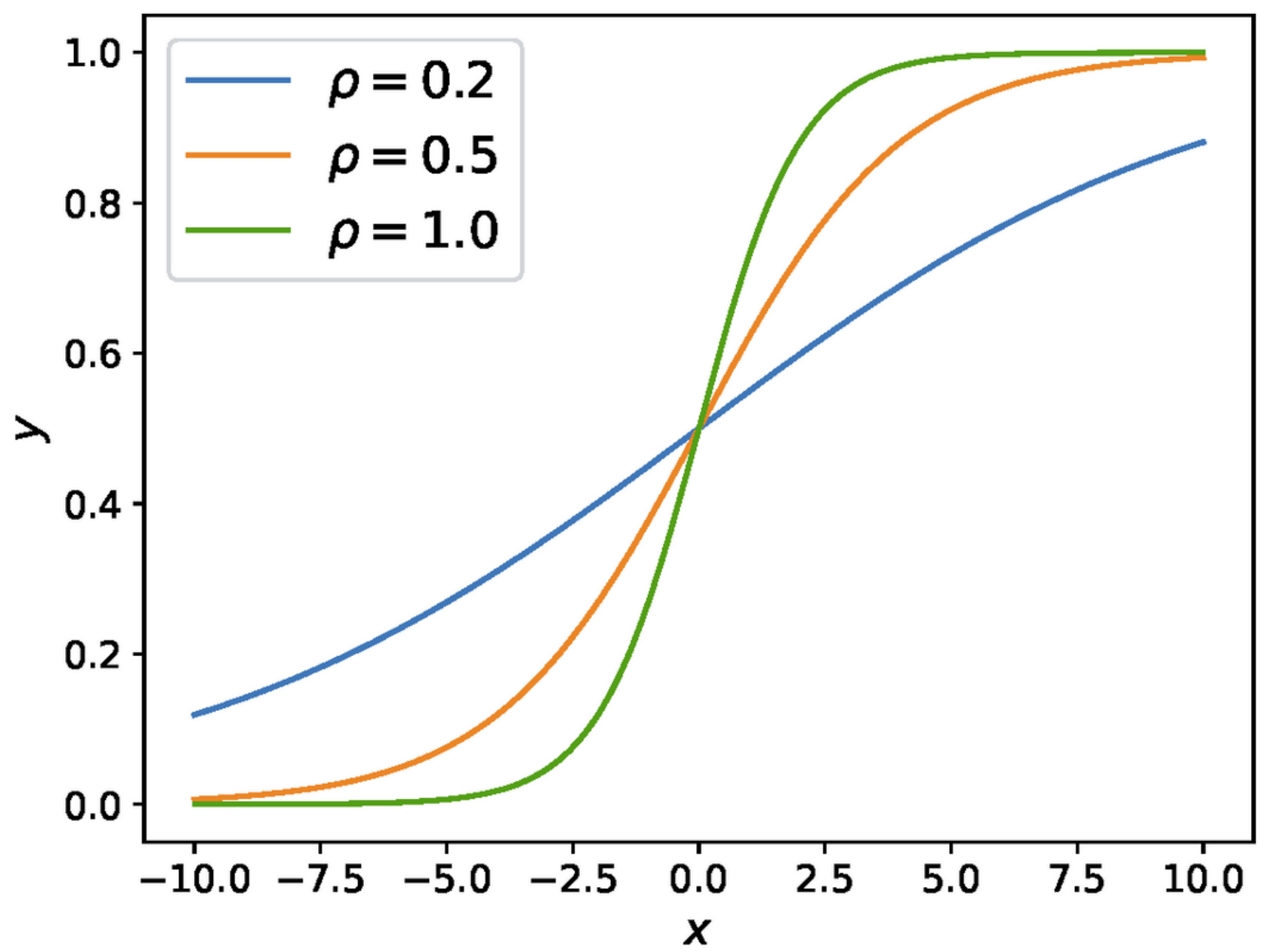

Figure 4

Graph of sigmoid with different coeffcient. 


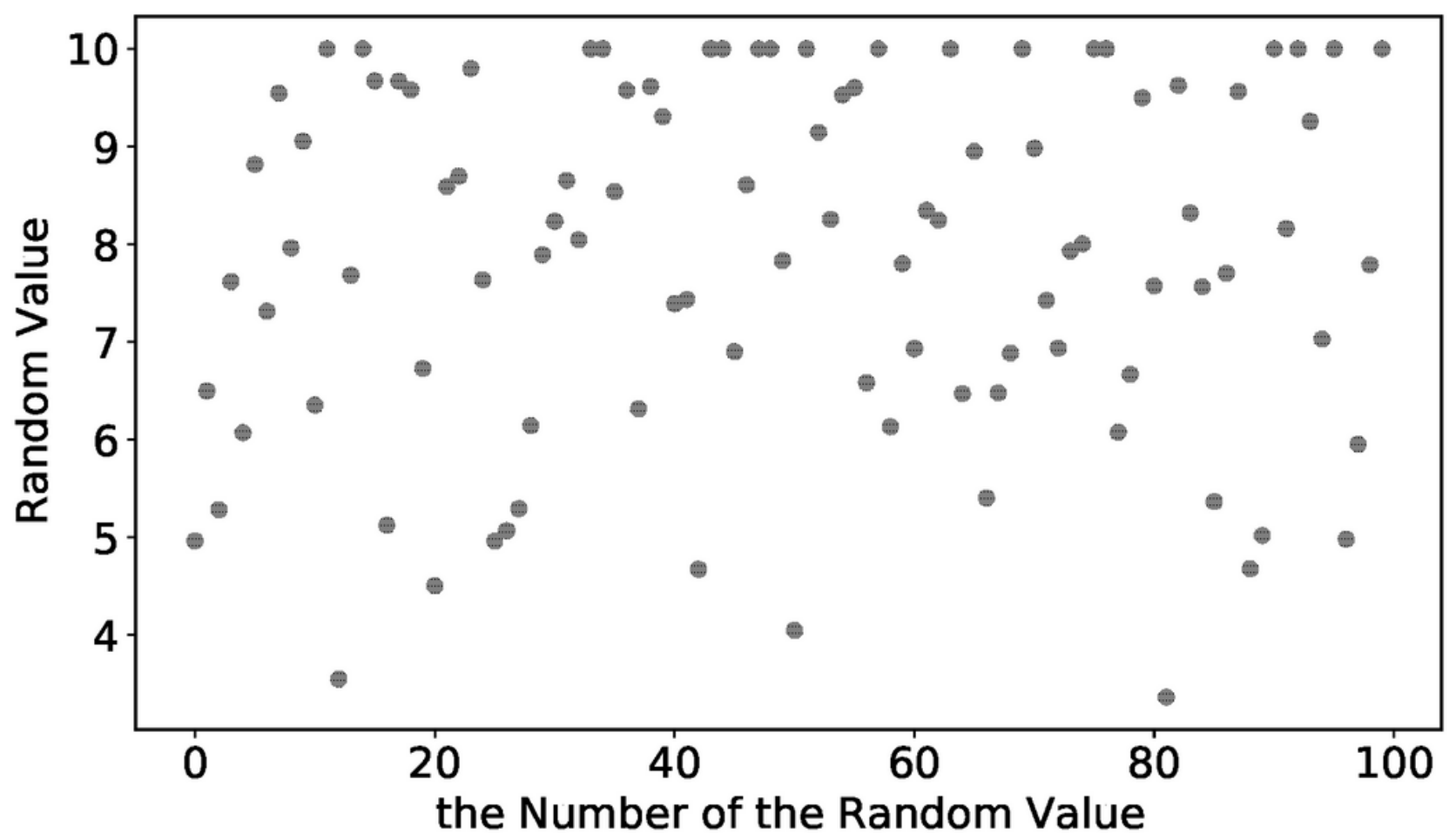

Figure 5

The first 100 values of the quality of the task results. 


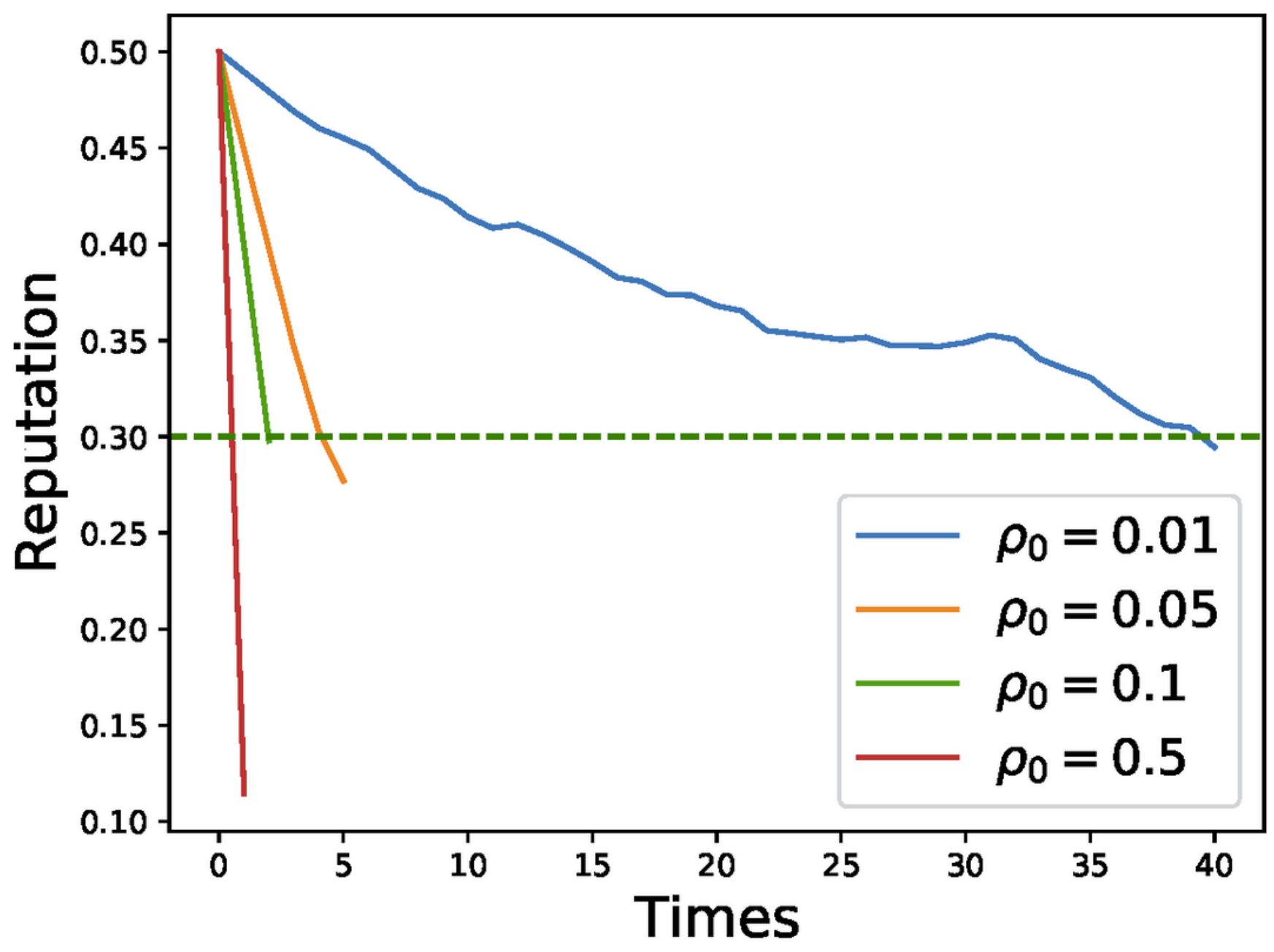

Figure 6

The number of transactions for reputation values drop from 0.5 to 0.3 . 


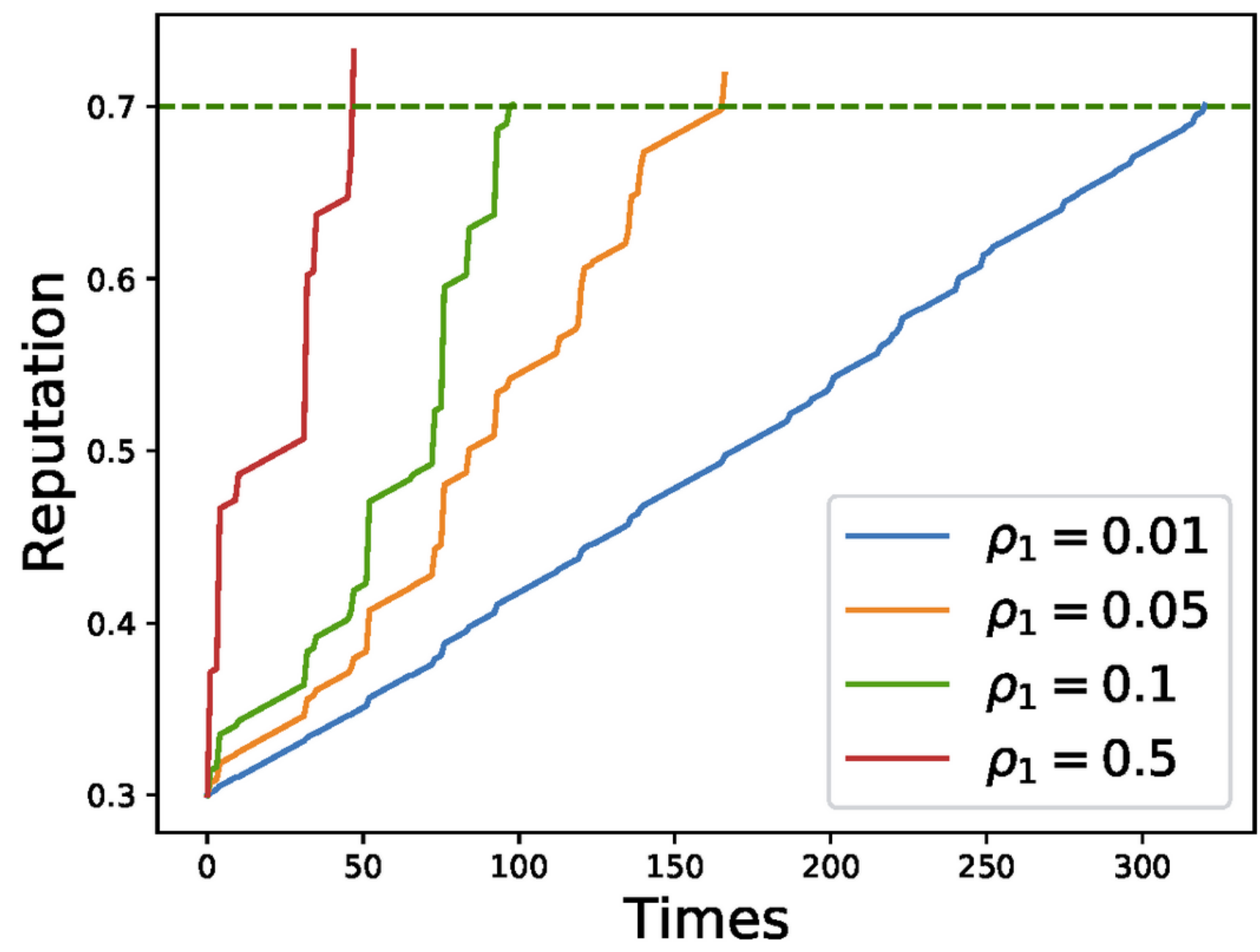

Figure 7

The number of transactions for reputation values raise from 0.3 to 0.7 . 


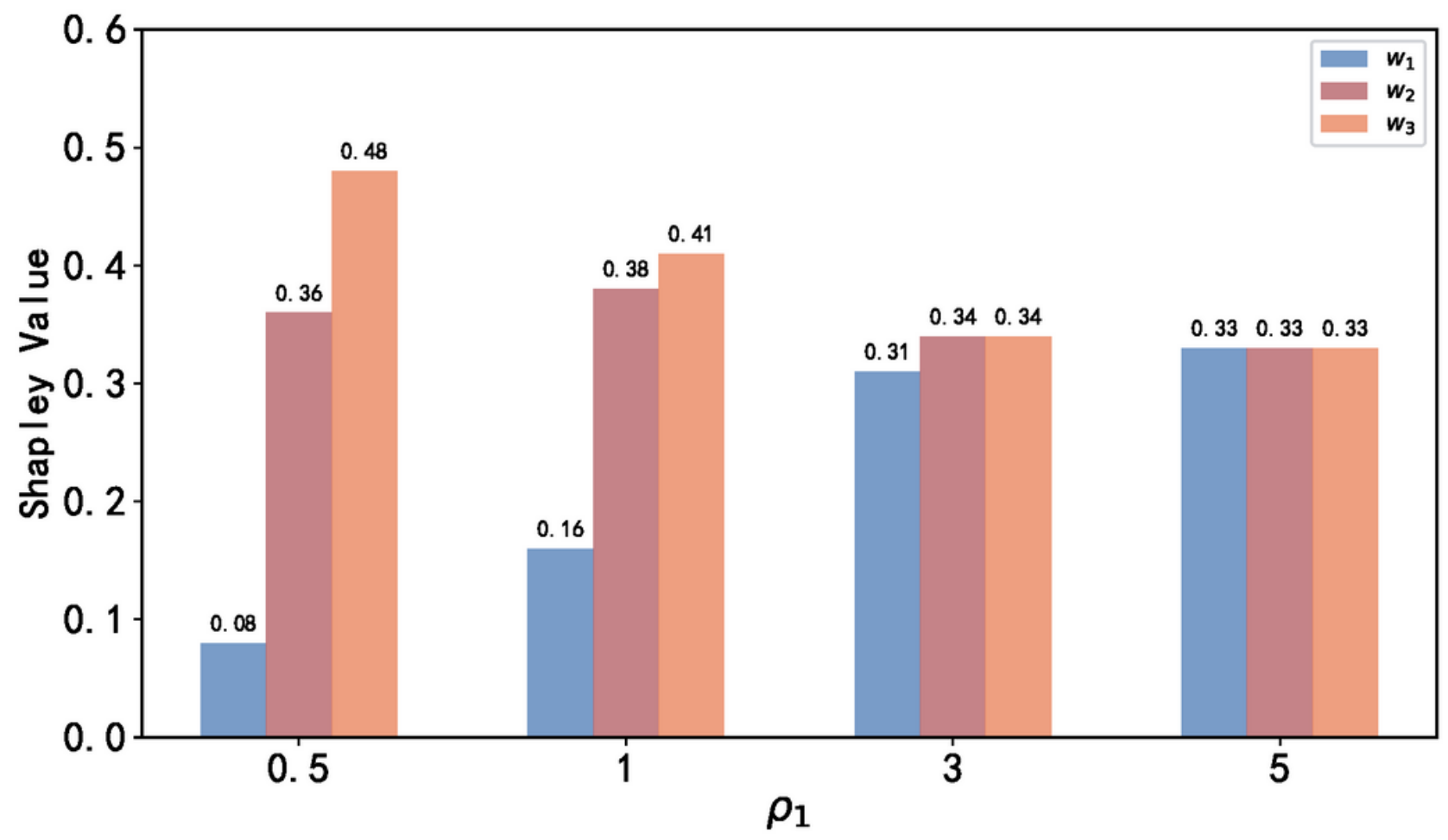

Figure 8

Workers' shapley values. 